Pacific Journal of Mathematics

EXISTENCE OF SPECIAL $K$-SETS IN CERTAIN LOCALLY 


\title{
EXISTENCE OF SPECIAL $K$-SETS IN CERTAIN LOCALLY COMPACT ABELIAN GROUPS
}

\author{
Frank B. Miles
}

\begin{abstract}
In all that follows, $G$ is an infinite, nondiscrete, locally compact $T_{0}$ abelian group with character group $X$ and $\Delta$ is a nonempty subset of $X$. In a standard proof of the existence of infinite (in fact, perfect) Helson sets (see for example Hewitt and Ross) it is shown that each nonvoid open subset of an arbitrary $G$ contains a $K$-set (terminology of Hewitt and Ross) homeomorphic to Cantor's ternary set (or, in the terminology of Rudin, a Kronecker set or a set of type $K_{a}$ homeomorphic to the Cantor set). In this paper, it is shown that $K_{0,4}$-sets or $K_{a, 4}$-sets homeomorphic to the Cantor set exist in profusion in a large class of infinite nondiscrete locally compact $T_{0}$ abelian groups $G$, provided that $\bar{\Delta}$ is not compact. (A nonvoid subset $E$ of $G$ is called a $K_{0,4}$-set if for every continuous function from $E$ to $\mathrm{T}$, the circle group, and every $\varepsilon>0$, there is a $\gamma \in \Delta$ such that $|\gamma(x)-f(x)|<\varepsilon$ for all $x \in E$. Let $a$ be an integer greater than one. $A$ nonvoid subset $E$ of $G$ is called a $K_{a, s}$-set if it is totally disconnected and every continuous function on $E$ with values in the set of $a$ th roots of unity is the restriction to $E$ of some $\gamma \in \Delta$.)
\end{abstract}

The following theorems will be proved.

Theorem I. Let $G$ be compact. Let $\Delta$ be infinite. Suppose that, except for the character which is identically $1, \Delta \Delta^{-1}$ consists solely of elements of infinite order. (This condition is satisfied automatically if $G$ is connected, for then $X$ is torsion-free.) Then every nonvoid open set in $G$ contains a $K_{0,4}$-set homeomorphic to the Cantor set.

THEOREM II. Let $G$ be locally connected. Suppose that $\bar{\Delta}$ is not compact. Then every nonvoid open set in $G$ contains a $K_{0,4}$-set homeomorphic to the Cantor set.

THEOREM III. Let $G$ be a compact torsion group. Let $A$ be infinite. Then there is an integer $a \geqq 2$ such that every nonvoid open set in $G$ contains a translate of a $K_{a, 4}$-set homeomorphic to the Cantor set.

1. Preliminaries.

Notation 1.1. We denote Haar measure on $G$ by $m$, with $m(G)=1$ when $G$ is compact. When $H$ is a subgroup of $G$, we write 
$\left.\Delta\right|_{H}$ for $\left\{\left.\gamma\right|_{H}: \gamma \in \Delta\right\} . M(P)$ denotes the set of all (finite) regular Borel measures on the compact subset $P$ of $G$.

$C(A, B)$ denotes the set of all continuous functions from $A$ to $B$, where $A$ and $B$ are topological spaces. If $B=\mathrm{C}$, the set of complex numbers, we write $C(A)$ instead of $C(A, \mathrm{C})$.

$\mathbf{Z}$ is the group of integers. $\mathbf{R}$ is the group of real numbers. $\mathbf{Q}$ is the (discrete) group of rational numbers. $\mathbf{N}$ is the set of positive integers. When $a$ is an integer greater than one, $\mathbf{Z}_{a}$ is the additive group of integers modulo $a$ and $\mathbf{T}_{(a)}$ is the multiplicative group of $a$ th roots of unity.

1 is the identity element of $X$.

$\Pi_{\iota \in I}^{*} G_{\iota}$ is the weak direct product of the groups $G_{\iota}$.

REMARKS 1.2.

(a) In $\S 5$, we give examples which show some of the limitations of Theorems I, II, and III.

(b) The hypothesis on $\Delta \Delta^{-1}$ in Theorem I is related to connectedness, as will be shown in Theorem 2.1.

(c) When $G$ is compact, a $K_{0,4}$-set (or $K_{a, 4}$-set) $E$ is a 4 -Helson set-i.e., a set with the property that every $f \in C(E)$ has the form $f=\left.\check{g}\right|_{E}$ for some $g \in L_{1}(X)$ which vanishes off $\Delta$. When $G$ is not compact, a $K_{0,4}$-set need not be a $\Delta$-Helson set as the example $G=$ $X=\mathbf{R}$ and $\Delta=\mathbf{Q}$ shows.

(d) Our proof of Theorem II for the case where $G$ is metrizable uses a technique due to Kaufman, [6, p. 184-185 and 7]. The general case follows from the case where $G$ is metrizable and from Theorem I. Our proofs of Theorems I and III depend on the notion of an equidistributed sequence in a compact group. This notion for the case $G=\mathbf{T}$ is due to Weyl [9]. The notion has been generalized by Eckmann [2] and Hlawka [5]. Eckmann's work offers more than enough generality for our purposes; relevant parts are given below in 1.3 and 1.4 .

DEFINITION 1.3. Let $H$ be a compact abelian group with Haar measure $\mu$ and $\mu(H)=1$. Let $\left\{\alpha_{j}\right\}_{j=1}^{\infty}$ be a sequence in $H$. For $F \subset H$, let $n(F)$ be the number of $\alpha_{j}$ with index $j \leqq n$ which are in $F$. The sequence $\left\{a_{j}\right\}_{j=1}^{\infty}$ is said to be equidistributed in $H$ if $\lim _{n \rightarrow \infty} n(F) / n=$ $\mu(F)$ for all closed $F$ with the property that $\mu$ (boundary $F$ ) $=0$.

THEOREM 1.4. Let $H$ be a compact abelian group with Haar measure $\mu$ and $\mu(H)=1$. Let $\left\{\alpha_{j}\right\}_{j=1}^{\infty}$ be a sequence in $H$. The following are equivalent:

(i) $\left\{\alpha_{j}\right\}_{j=1}^{\infty}$ is equidistributed in $H$; 
(ii) for every continuous character $\gamma$ of $H$ such that $\gamma \neq 1$, we have $\lim _{n \rightarrow \infty} n^{-1} \sum_{j=1}^{n} \gamma\left(\alpha_{j}\right)=0$.

\section{REMARKS 1.5 .}

(a) In the proofs of Theorems I and III we will use the equivalence of (i) and (ii) in Theorem 1.4 for the cases $H=\mathbf{T}$ and $H=\mathbf{T}_{(a)}$ respectively. If $H=\mathbf{T}$ we have Weyl's original result: The sequence $\left\{\alpha_{j}\right\}_{j=1}^{\infty} \subset \mathbf{T}$ is equidistributed in $\mathbf{T}$ if and only if $\lim _{n \rightarrow \infty} n^{-1} \sum_{j=1}^{n} \alpha_{j}^{r}=0$ for all nonzero integers $r$ (or, equivalently, for all $r \in \mathbf{N}$ ) [9]. If $H=\mathbf{T}_{(a)}$, we have: The sequence $\left\{\alpha_{j}\right\}_{j=1}^{\infty} \subset \mathbf{T}_{(a)}$ is equidistributed in $\mathbf{T}_{(a)}$ if and only if for every integer $r \in\{1,2, \cdots, a-1\}$ we have $\lim _{n \rightarrow \infty} n^{-1} \sum_{j=1}^{n} \alpha_{j}^{r}=0$.

(b) Eckmann's definition differs from Definition 1.3 in that he omits the restriction $\mu$ (boundary $F$ ) $=0$. This restriction is necessary, as has been pointed out [3].

\section{Proof of Theorem I.}

2.1. We first investigate the hypothesis on $\Delta \Delta^{-1}$ in the statement of Theorem I and find that it is related to connectedness.

THEOREM. Let $G$ be compact. Let $\Delta$ be a countably infinite subset of $X$. The following are equivalent:

(i) $\Delta \Delta^{-1} \backslash\{1\}$ consists solely of elements of infinite order;

(ii) $G$ contains a compact connected metrizable subgroup $H$ with the property that $\left.\delta \rightarrow \delta\right|_{H}$ is a one-to-one map from $\Delta$ to the character group of $H$.

Proof. (ii) implies (i): Let $\delta_{1}$ and $\delta_{2}$ be distinct elements of $\Delta$. Then $\left.\delta_{1}\right|_{I I} \neq\left.\delta_{2}\right|_{H}$, so $\left.\delta_{1} \delta_{2}^{-1}\right|_{H} \neq 1$. Since $H$ is connected, its character group is torsion-free. Hence, $\left.\delta_{1} \delta_{2}^{-1}\right|_{H}$ has infinite order and therefore so does $\delta_{1} \delta_{2}^{-1}$.

(i) implies (ii): Let $\Gamma$ be a maximal torsion-free independent subset of $\Delta$. (Clearly, $\Delta$ contains at most one element of finite order, so $\Gamma$ is nonvoid.) We have $\Gamma=\left\{\gamma_{1}, \cdots, \gamma_{p}\right\}$ for some positive integer $p$ or $\Gamma=\left\{\gamma_{1}, \gamma_{2}, \cdots\right\}$. If $\Gamma$ is finite, let $P=\mathbf{Q}^{p}$. If not, let $P$ be the weak direct product of countably many copies of $\mathbf{Q}$. (In either case, $P$ is countable.) For $n \in \mathbf{N}$ (and $n \leqq p$ if $\Gamma$ is finite) let $e_{n}$ be that element of $P$ with $n$th coordinate equal to 1 and all other coordinates equal to zero. Let $Y$ be the subgroup of $X$ generated by $\Gamma$. Since $\Gamma$ is independent, the map $\gamma_{n} \rightarrow e_{n}$ extends to a (one-to-one) homomorphism from $Y$ to $P$. Since $P$ is divisible, this homomorphism extends to a homomorphism $\phi: X \rightarrow P$. Hence $W=X / \operatorname{ker} \phi$ is isomorphic to a subgroup of $P$. Let $H$ be the annihilator of ker $\phi$ in $G$. Then $H$ 
is a closed subgroup of $G$ and has character group $W$, which is torsion-free and countable. Hence, $H$ is connected and metrizable. Now $\left.\delta_{1}\right|_{H}=\left.\delta_{2}\right|_{H}$ if and only if $\delta_{1} \delta_{2}^{-1} \in \operatorname{ker} \phi$. Let $\delta_{1}$ and $\delta_{2}$ be distinct elements of $\Delta$. It is sufficient to show that $\delta_{1} \delta_{2}^{-1} \notin \operatorname{ker} \phi$. Since $\Gamma$ is a maximal torsion-free independent subset of $\Delta$, there exist nonzero integers $r_{1}$ and $r_{2}$ such that $\delta_{1}^{r_{1}}$ and $\delta_{2}^{r_{2}}$ are in $Y$. Therefore there is a nonzero integer $r$ such that $\left(\delta_{1} \delta_{2}^{-1}\right)^{r} \in Y$. By the hypothesis on $\Delta \Delta^{-1}$, we have $\left(\delta_{1} \delta_{2}^{-1}\right)^{r} \neq 1$. Since $\phi$ is one-to-one on $Y, r \phi\left(\delta_{1} \delta_{2}^{-1}\right)=\phi\left(\left(\delta_{1} \delta_{2}^{-1}\right)^{r}\right)$ is not the identity of $P$. Hence $\delta_{1} \delta_{2}^{-1} \notin \operatorname{ker} \phi$ and the proof is complete.

LEMmA 2.2. Let $G$ be compact. Let $\Delta=\left\{\gamma_{1}, \gamma_{2}, \cdots\right\}$ be a countably infinite set of distinct elements of $X$ arranged in any fixed order. Suppose that $\Delta \Delta^{-1} \backslash\{1\}$ consists solely of elements of infinite order. Then for m-almost all $x \in G$, the sequence $\left\{\gamma_{j}(x)\right\}_{j=1}^{\infty}$ is equidistributed in $\mathbf{T}$.

Proof. Our proof follows Weyl [9]. For $x \in G, n \in \mathbf{N}$, and $r \in \mathbf{N}$, define $f_{n r}(x)=n^{-1} \sum_{j=1}^{n} \gamma_{j}^{r}(x)$. From our hypothesis on $\Delta \Delta^{-1}$ we find that $\gamma_{j}^{r} \overline{\gamma_{k}^{r}}=1$ implies that $\gamma_{j}=\gamma_{k}$. Since $G$ is compact, $\int_{G} \gamma(x) d m(x)=$ 0 when $\gamma \neq 1$. Thus, we have

$$
\int_{G}\left|f_{n r}\right|^{2} d m=n^{-2} \int_{G} \sum_{j, k=1}^{n} \gamma_{j}^{r}(x) \overline{\gamma_{k}^{r}(x)} d m(x)=n^{-1} .
$$

Therefore we have $\sum_{n=1}^{\infty}\left\|f_{n^{2}, r}\right\|_{2}^{2}<\infty$ and hence $f_{n^{2}, r}(x) \rightarrow 0$ as $n \rightarrow \infty$ for $m$-almost all $x \in G$. Suppose that $f_{n^{2}, r}(x) \rightarrow 0$ as $n \rightarrow \infty$ for all $x \notin A_{r}$ where $m\left(A_{r}\right)=0$.

For $n \in \mathbf{N}$, let $\lambda(n)$ be the positive integer such that $\lambda^{2} \leqq n<$ $(\lambda+1)^{2}$. Then we have $\left|n f_{n r}(x)-\lambda^{2} f_{\lambda^{2}, r}(x)\right| \leqq 2 \lambda$ and hence

$$
\left|f_{n r}(x)-\frac{\lambda^{2}}{n} f_{\lambda^{2}, r}(x)\right| \leqq 2 / \sqrt{n} \text {. }
$$

Let $\varepsilon>0$. Fix $x \notin A_{r}$. Then there is a positive integer $M$ such that $\left|f_{\lambda^{2}, r}(x)\right|<\varepsilon / 2$ whenever $\lambda \geqq M$. Let $n \geqq M^{2}$ and $n>16 / \varepsilon^{2}$. Let $\lambda$ be such that $\lambda^{2} \leqq n<(\lambda+1)^{2}$. Then $\lambda^{2} / n \leqq 1,2 / \sqrt{n}<\varepsilon / 2$, and $\lambda^{2} \geqq$ $M^{2}$, so we have

$$
\left|f_{n r}(x)\right| \leqq\left|f_{n r}(x)-\frac{\lambda^{2}}{n} f_{\lambda^{2}, r}(x)\right|+\frac{\lambda^{2}}{n}\left|f_{\lambda^{2}, r}(x)\right|<2 / \sqrt{n}+\varepsilon / 2<\varepsilon .
$$

Hence, $f_{n r}(x) \rightarrow 0$ as $n \rightarrow \infty$ for all $x \notin A_{r}$.

Let $A=\cup A_{r}$. Then $m(A)=0$ and for $x \notin A$ we have for all $r \in \mathbf{N}$ that $f_{n r}(x) \rightarrow 0$ as $n \rightarrow \infty$. Therefore, by $1.5(a),\left\{\gamma_{j}(x)\right\}_{j=1}^{\infty}$ is equidistributed in $\mathbf{T}$ for all $x \notin A$. 
LEMma 2.3. Let $G$ and $\Delta$ be as in Theorem 1. Let $V_{1}, \cdots, V_{k}$ be nonvoid open subsets of $G$. Then there exist $x_{j} \in V_{j}(1 \leqq j \leqq k)$ with the property that for every $\varepsilon>0$ and for all $z_{1}, \cdots, z_{k} \in \mathbf{T}$ there is a $\gamma \in \Delta$ such that $\left|\gamma\left(x_{j}\right)-z_{j}\right|<\varepsilon(1 \leqq j \leqq k)$, i.e., there exist $x_{j} \in V_{j}$ $(1 \leqq j \leqq k)$ such that $\left\{x_{1}, \cdots, x_{k}\right\}$ is a $K_{0,4}$-set.

Proof. We may suppose that $\Delta$ is countable. Let $q \in\{1,2, \cdots, k\}$. Let " $P(q)$ holds for $x_{1}, \cdots, x_{q}$ " mean " $x_{j} \in V_{j}(1 \leqq j \leqq q)$ and $\left\{x_{1}, \cdots, x_{q}\right\}$ is a $K_{0,4}$ set." By Lemma 2.2, there is an $x_{1} \in V_{1}$ such that $P(1)$ holds for $x_{1}$. Suppose that $1 \leqq r \leqq k-1$ and that $P(r)$ holds for $x_{1}, \cdots, x_{r}$. It is sufficient to show there is an $x_{r+1} \in V_{r+1}$ such that $\left.P^{\prime} r+1\right)$ holds for $x_{1}, \cdots, x_{r+1}$. Let $A=\left\{w \in V_{r+1} \mid P(r+1)\right.$ does not hold for $x_{1}, \cdots$, $\left.x_{r}, w\right\}$. It is sufficient to show that $m(A)=0$. Let $S$ be a countable dense subset of T. Then $w \in A$ if and only if $w \in V_{r+1}$ and there exist $p \in \mathbf{N}$ and $s_{1}, \cdots, s_{r+1} \in S$ such that for all $\gamma \in \Delta$ either $\left|\gamma\left(x_{j}\right)-s_{j}\right| \geqq p^{-1}$ for some $j(1 \leqq j \leqq r)$ or $\left|\gamma(w)-s_{r+1}\right| \geqq p^{-1}$, i.e., we have

$$
A=\bigcup_{p \in \mathrm{N}} \bigcup_{s_{1} \in S} \cdots \bigcup_{s_{r+1} \in S} A\left(p, s_{1}, \cdots, s_{r+1}\right)
$$

where $A\left(p, s_{1}, \cdots, s_{r+1}\right)=\bigcap_{r \in \Delta}\left\{y \in V_{r+1}:\left|\gamma(y)-s_{r+1}\right| \geqq p^{-1}\right.$ or at least one $\left.\left|\gamma\left(x_{j}\right)-s_{j}\right| \geqq p^{-1}\right\}$.

Let

$$
\widetilde{\Delta}\left(p, s_{1}, \cdots, s_{r}\right)=\left\{\gamma \in \Delta:\left|\gamma\left(x_{j}\right)-s_{j}\right|<p^{-1}, 1 \leqq j \leqq r\right\} .
$$

Then we have

$$
\begin{aligned}
& A\left(p, s_{1}, \cdots, s_{r+1}\right) \\
= & \left\{y \in V_{r+1}:\left|\gamma(y)-s_{r+1}\right| \geqq p^{-1} \text { for all } \gamma \in \widetilde{J}\left(p, s_{1}, \cdots, s_{r}\right)\right\} .
\end{aligned}
$$

Hence, it is sufficient to show that each $\widetilde{\Delta}\left(p, s_{1}, \cdots, s_{r}\right)$ is infinite (for then, by Lemma 2.2 , each $A\left(p, s_{1}, \cdots, s_{r+1}\right)$ is $m$-null and therefore so is $A$ ).

We assume that for some $p \in \mathbf{N}$ and $s_{1}, \cdots, s_{r} \in S$ the set $\widetilde{\Delta}=$ $\widetilde{\Delta}\left(p, s_{1}, \cdots, s_{r}\right)$ is finite and use this to obtain a contradiction. A basic neighborhood of the point $\mathbf{z}=\left(z_{1}, \cdots, z_{r}\right) \in \mathbf{T}^{r}$ has the form $B(\mathbf{z}, \varepsilon)=$ $\left\{\mathbf{w}=\left(w_{1}, \cdots, w_{r}\right):\left|z_{j}-w_{j}\right|<\varepsilon, 1 \leqq j \leqq r\right\}$ for some $\varepsilon>0$. Let $\mathbf{s}=$ $\left(s_{1}, \cdots, s_{r}\right)$ and $\mathbf{x}=\left(x_{1}, \cdots, x_{r}\right)$. For $\gamma \in \Delta$, let $\gamma(\mathbf{x})=\left(\gamma\left(x_{1}\right), \cdots, \gamma\left(x_{r}\right)\right)$. If $\tilde{\Delta}$ is finite, then $\left\{\gamma \in \Delta \mid \gamma(\mathbf{x}) \in B\left(\mathbf{s}, p^{-1}\right)\right\}$ is finite. Then there exist $\mathbf{z} \in B\left(\mathbf{s}, p^{-1}\right)$ and $\varepsilon>0$ be such that $B(\mathbf{z}, \varepsilon) \subset B\left(\mathbf{s}, p^{-1}\right)$ and $B(\mathbf{z}, \varepsilon)$ is disjoint from $\{\gamma(\mathbf{x}) \mid \gamma \in \Delta\}$. This contradicts the induction hypothesis that $P(r)$ holds for $x_{1}, \cdots, x_{r}$.

THEOREM 2.4. Theorem I holds when $G$ is metrizable.

Proof. Repeat the proof of $[4,(41.5)$, part I] choosing all charac- 
ters in $\Delta$ and using Lemma 2.3 whenever [4] uses [4, (41.3)].

TheOREM 2.5. Let $G$ and $\triangle$ be as in Theorem $I$. Let $U$ be a neighborhood of the identity in $G$. Then $U$ contains a $K_{0,4}$-set homeomorphic to the Cantor set.

Proof. By Theorem 2.1, $G$ contains a compact connected metrizable subgroup $H$ with the property that $\Gamma=\left.\Delta\right|_{I}$ is infinite. Let $V=U \cap H$. Since $H$ is connected, its character group is torsion-free. Hence, by Theorem 2.4, $V$ contains a $K_{0, r}$-set $P$ homeomorphic to the Cantor set. Clearly, $P$ is a $K_{0,4}$-set contained in $U$.

THEOREM ${ }^{1}$ 2.6. Let $P$ be a compact metrizable $K_{0,4}$-set in $G$, where $G$ is compact and $\Delta \Delta^{-1} \backslash\{\mathbf{1}\}$ consists solely of elements of infinite order. Then for almost all $x \in G, x P$ is a $K_{0,4}$-set.

Proof. Let $\left\{f_{1}, f_{2}, \cdots\right\}$ be a (uniformly) dense subset of $C(P, \mathbf{T})$. For each $j$, there is a sequence $\left\{\gamma_{i j}\right\}_{i=1}^{\infty}$ of elements of $\Delta$ such that $\gamma_{i j} \rightarrow f_{j}$ uniformly on $P$. By Lemma 2.2 , there is an $m$-null set $A_{j}$ such that $\left\{\gamma_{i j}(x)\right\}_{i=1}^{\infty}$ is equidistributed in $\mathbf{T}$ whenever $x \in G \backslash A_{j}$. Let $A=\cup A_{j}$. Then $A$ is $m$-null. Let $x \in G \backslash A$. For each $j$, let $g_{j}(x y)=$ $f_{j}(y)$. To show that $x P$ is a $K_{0,4}$-set, it is sufficient to show that each $g_{j}$ is uniformly approximable by $\left\{\gamma_{i j}: i, j=1,2, \cdots\right\}$. Let $\varepsilon>0$. Fix $j$. Then for some $i_{0}$, we have $\left|\gamma_{i j}(y)-f_{j}(y)\right|<\varepsilon / 2$ for all $y \in P$ whenever $i>i_{0}$ and, since $\left\{\gamma_{i j}(x)\right\}_{i=1}^{\infty}$ is equidistributed in $\mathbf{T}$, there is an $i>i_{0}$ such that $\left|\gamma_{i j}(x)-1\right|<\varepsilon / 2$. For this $i$ we have $\mid \gamma_{i j}(x y)-$ $g_{j}(x y) \mid<\varepsilon$ for all $y \in P$.

Proof of Theorem I. 2.7. Immediate from Theorems 2.5 and Theorem 2.6.

\section{Proof of Theorem II.}

THEOREM 3.1. Let $G$ be locally connected. Let $\Delta$ be such that $\bar{J}$ is not compact. Let $U$ be a neighborhood of the identity in G. Then there is a $\gamma$ in $\Delta$ such that $\gamma(U)=\mathbf{T}$.

Proof. The topology on $X$ is the restriction of the compact-open topology on $C(G)$ to the (closed) subspace $X$ of $C(G)$. Hence, $\bar{\Delta}$ is

${ }^{1}$ In the original version of this paper, the conclusion of Theorem I was as follows: Every open set in $G$ containing an element of finite order contains a $K_{0,4}$-set homeomorphic to the Cantor set and, if $G$ is metrizable, every nonvoid open set in $G$ contains a $K_{0,4}$-set homeomorphic to the Cantor set. Theorem 2.6 and the stronger version of Theorem I which it yields are due to Robert Kaufman [private communication, December, 1971]. 
compact as a subspace of $X$ if and only if it is compact as a subspace of $C(G)$ with the compact-open topology. Since by hypothesis $\bar{\Delta}$ is not compact, it follows from Ascoli's Theorem that $\Delta$ is not equicontinuous [1, p. 267] and, hence, that $\Delta$ is not equicontinuous at the identity of $G$. Therefore, there exists $\varepsilon>0$ such that for every neighborhood $W$ of the identity in $G$, there is an $x \in W$ and a $\gamma \in \Delta$ such that $|\gamma(x)-1| \geqq \varepsilon$. Let $S=\left\{e^{i t} \mid 0 \leqq t \leqq \varepsilon / 2\right\}$. Let $M$ be a positive integer with the property that $S^{H}=\mathbf{T}$. Let $V$ be a connected neighborhood of the identity in $G$ such that $V^{M} \subset U$. Then there exist $x \in V$ and $\gamma \in \Delta$ such that $|\gamma(x)-1| \geqq \varepsilon$. Hence, $\gamma(V)$ contains an arc of length at least $\varepsilon$. Therefore we have $\mathbf{T}=\gamma(V)^{M} \subset \gamma(U) \subset \mathbf{T}$.

THEOREM 3.2. Let $G$ be locally connected and metrizable. Let $\triangle$ be such that $\bar{J}$ is not compact. Let $E$ be a compact totally disconnected subset of $\mathbf{R}$ or $\mathbf{T}$. Then there is a first category set $H \subset C(E, G)$ such that each $f \in C(E, G) \backslash H$ maps $E$ homeomorphically onto a $K_{0,4}$-set in $G$.

Proof. Our proof follows the ideas of Kaufman [7] as given by Katznelson [6, p. 184-185].

For $h \in C(E, \mathbf{T}), f \in C(E, G)$, and $\varepsilon>0$, let "(*) holds for $h, f$ and $\varepsilon$ " mean "there is a $\gamma \in \Delta$ such that $|\gamma(f(y))-h(y)|<\varepsilon$ for all $y \in E_{\text {。" }}$ Let $f \in C(E, G)$. Clearly, $f$ is a homeomorphism of $E$ onto $f(E)$ if and only if $f$ is one-to-one. Also, if $f$ is not one-to-one, it is clear that there exist $h \in C(E, \mathbf{T})$ and $\varepsilon>0$ such that $\left(^{*}\right)$ fails for $h, f$, and $\varepsilon$. Hence, $f$ is a homeomorphism of $E$ onto $f(E)$ and $f(E)$ is a $K_{0,4}$-set if and only if for every $h \in C(E, T)$ and every $\varepsilon>0,\left(^{*}\right)$ holds for $h, f$, and $\varepsilon$ 。

Let $d$ be an invariant metric on $G$ compatible with the topology of $G$. For $f$ and $g$ in $C(E, G)$, let $D(f, g)=\sup \{d(f(y), g(y)) \mid y \in E\}$ 。 Observe that $D(f, g)<\infty$ since $E$ is compact.

Let $h \in C(E, \mathbf{T}), g \in C(E, G), \varepsilon>0$, and $\eta>0$. We now show that there exist an $f \in C(E, G)$ such that $\left(^{*}\right)$ holds for $h, f$, and $\varepsilon$ and $D(f, g)<\eta_{\text {. Let }} U$ ba the open $\eta$-ball about the identity $e$ of $G$. By Theorem 3.1, there is a $\gamma \in \Delta$ such that $\gamma(U)=\mathbf{T}$. Write $E=\bigcup_{j=1}^{n} E_{j}$, where the $E_{j}$ are disjoint nonvoid open-closed subsets of $E$ and $\gamma \circ g$ and $h$ both vary by less than $\varepsilon / 3$ on each $E_{j}$. (The $E_{j}$ exist since $E$ is totally disconnected.) Let $y_{j} \in E_{j}$ and suppose that $\gamma\left(g\left(y_{j}\right)\right)=\alpha_{j}$ and $h\left(y_{j}\right)=\beta_{j}, 1 \leqq j \leqq n$. Let $x_{j} \in U$ be such that $\gamma\left(x_{j}\right)=\overline{\alpha_{j}^{\prime}} \beta_{j}$. Define $f \in C(E, G)$ by $f(y)=x_{j} g(y)$ when $y \in E_{j}$. We see that $D(f, g)=$ $\max \left\{d\left(x_{j}, e\right)\right\}<\eta$ and for $y \in E_{j}$ we have

$$
\begin{aligned}
& |\gamma(f(y))-h(y)| \leqq\left|\gamma(g(y)) \gamma\left(a_{j}\right)-\gamma\left(g\left(y_{j}\right)\right) \gamma\left(x_{j}\right)\right| \\
& \quad+\left|\gamma\left(g\left(y_{j}\right)\right) \gamma\left(x_{j}\right)-h\left(y_{j}\right)\right|+\left|h\left(y_{j}\right)-h(y)\right|<\frac{\varepsilon}{3}+0+\frac{\varepsilon}{3}<\varepsilon .
\end{aligned}
$$


Hence, $\left({ }^{*}\right)$ holds for $h, f$, and $\varepsilon$.

For $h \in C(E, \mathbf{T})$ and $\varepsilon>0$, let $H(h, \varepsilon)=\left\{f \in C(E, G) \mid\right.$ (*) fails for $^{*}$ $h, f$, and $\varepsilon\}$. It is easy to show that $H(h, \varepsilon)$ is closed. By the preceding paragraph, $H(h, \varepsilon)$ is nowhere dense in $C(E, G)$. Let $\left\{h_{n}\right\}_{n=1}^{\infty}$ be dense in $C(E, \mathbf{T})$. Let $H=\cup_{n, k=1}^{\infty} H\left(h_{n}, 1 / k\right)$. Then $H$ is a first category set in the complete metric space $C(E, G)$. Also, we have $f \in C(E, G) \backslash H$ if and only if every $h \in C(E, \mathbf{T})$ can be uniformly approximated by $\gamma \circ f$ 's $(\gamma \in \Delta)$, which by the second paragraph of the proof is true if and only if $f$ is a homeomorphism and $f(E)$ is a $K_{0,4}$-set.

THEOREM 3.3. Theorem II holds when $G$ is metrizable.

Proof. Let $U$ be a nonvoid open subset of $G$. Let $E$ be the Cantor set. Let $H$ be as in Theorem 3.2. The result follows from Theorem 3.2 since $C(E, U)$ is open in $C(E, G)$ and $C(E, G) \backslash H$ is dense in $C(E, G)$.

THEOREM 3.4. Let $G$ be locally connected. Then $G$ is topologically isomorphic with $D \times \mathbf{R}^{n} \times K$, where $D$ is discrete, $n$ is a nonnegative integer, and $K$ is a compact, connected, locally connected abelian group.

Proof. Let $C$ be the component of the identity in $G$. Then $G$ is topologically isomorphic with $(G / C) \times C$. Since $G / C$ is totally disconnected and locally connected, it is discrete. Since $C$ is connected and locally connected, it is topologically isomorphic with $\mathbf{R}^{n} \times K$, where $n$ is a nonnegative integer and $K$ is compact, connected, and locally connected.

Proof of Theorem II. 3.5. By Theorem 3.4, we may suppose that $G=H \times K$, where $H$ is locally connected and metrizable and $K$ is compact, connected, and locally connected. We then have $X=$ $Y \times F$, where $Y$ and $F$ are the character groups of $H$ and $K$, respectively. Let $U$ be a nonvoid open subset of $G$. We may suppose that $U=V \times W$, where $V$ and $W$ are nonvoid open subsets of $H$ and $K$, respectively. We denote elements of $X$ by $(\alpha, \beta)$, where $\alpha \in Y$ and $\beta \in F$. Let $\Gamma=\{\beta \in F \mid(\alpha, \beta) \in \Delta\}$.

Case 1. $\Gamma$ is finite: There is a $\beta_{0} \in \Gamma$ such that $\left\{\left(\alpha, \beta_{0}\right) \in \Delta\right\}^{-}$is not compact in $X$. Let $\Delta_{0}=\left\{\alpha \in Y \mid\left(\alpha, \beta_{0}\right) \in \Delta\right\}$. Then $\Delta_{0}^{-}$is not compact in $Y$. Hence, by Theorem 3.3, $V$ contains a $K_{0,1_{0}}$-set $P$ homeomorphic to the Cantor set. Let $z \in W$. Then $P \times\{z\}$ is a $K_{0,4}$-set in $U$ homeomorphic to the Cantor set. 
Case 2. $\quad \Gamma$ is infinite: Let $x \in V$. Let $\left\{\left(\alpha_{m}, \beta_{m}\right)\right\}_{m=1}^{\infty}$ be a sequence in $\Delta$ such that the $\beta_{m}$ are distinct and such that $\alpha_{m}(x) \rightarrow s \in \mathbf{T}$ as $m \rightarrow \infty$. Let $\Delta_{0}=\left\{\beta_{m}\right\}_{m=1}^{\infty}$. Since $\Delta_{0}$ is infinite and $K$ is compact and connected, $W$ contains a $K_{0, \Delta_{0}}$-set $P$ homeomorphic to the Cantor set by Theorem I. Then $\{x\} \times P$ is a $K_{0,4}$-set in $U$ homeomorphic to the Cantor set.

\section{Proof of Theorem III.}

Lemma 4.1. Let $k$ be an integer greater than one. Let $G$ be the product of infinitely many copies of $\mathbf{T}_{(k)}$. Let $\Delta$ be an infinite subset of $X$ and suppose there is an integer a greater than one such that all elements of $\triangle$ have order $a$ and that whenever $\gamma_{1}$ and $\gamma_{2}$ are distinct elements of $\Delta$, then $\gamma_{1} \gamma_{2}^{-1}$ has order $a$. Then for every sequence $\Delta_{0}=\left\{\gamma_{1}, \gamma_{2}, \cdots\right\}$ of distinct elements of $\Delta$, the sequence $\left\{\gamma_{j}(x)\right\}_{j=1}^{\infty}$ is equidistributed in $\mathbf{T}_{(a)}$ for m-almost all $x \in G$.

Proof. For $r \in\{1,2, \cdots, a-1\}$ and $n \in \mathbf{N}$, let $f_{n r}(x)=1 / n \sum_{j=1}^{n} \gamma_{j}^{r}(x)$. By our hypothesis on $\Delta, \gamma_{j} \neq \gamma_{l}$ implies that $\left(\gamma_{j} \gamma_{l}^{-1}\right)^{r} \neq 1$. Also, since $G$ is compact, $\int_{G} \gamma(x) d m(x)=0$ when $\gamma \neq 1$. Hence we have

$$
\int_{G}\left|f_{n r}\right|^{2} d m=n^{-2} \int_{G} \sum_{j, l=1}^{n} \gamma_{j}^{r}(x) \overline{\gamma_{l}^{r}(x)} d m(x)=n^{-1} \cdot
$$

We thus have $\sum_{n=1}^{\infty}\left\|f_{n^{2}, r}\right\|_{2}^{2}<\infty$ and hence $f_{n^{2}, r}(x) \rightarrow 0$ as $n \rightarrow \infty$ for $m$-almost all $x \in G$. Suppose that $f_{n^{2}, r}(x) \rightarrow 0$ as $n \rightarrow \infty$ for all $x \notin A_{r}$, where $m\left(A_{r}\right)=0$. The device used in the proof of Lemma 2.2 yields $f_{n r}(x) \rightarrow 0$ as $n \rightarrow \infty$ for $x \notin A_{r}$. Let $A=\cup_{r=1}^{a-1} A_{r}$. Then $m(A)=0$ and for $x \notin A$ we have for all $r \in\{1,2, \cdots, a-1\}$ that $f_{n r}(x) \rightarrow 0$ as $n \rightarrow \infty$. Therefore, by $1.5(a),\left\{\gamma_{j}(x)\right\}_{j=1}^{\infty}$ is equidistributed in $\mathbf{T}_{(a)}$ for all $x \notin A$.

Lemma 4.2. Let $k, G, \Delta$, and $a$ be as in Lemma 4.1. Let $V_{1}, \cdots, V_{n}$ be nonempty open subsets of $G$. Then there are $x_{j} \in V_{j}(1 \leqq$ $j \leqq n)$ such that $\left\{x_{1}, \cdots, x_{n}\right\}$ is a $K_{a, 4}$-set.

Proof. For a positive integer $q, y_{1}, \cdots, y_{q} \in \mathbf{T}_{(a)}$, and $w_{j} \in V_{j}(1 \leqq$ $j \leqq q)$, let $\Delta\left(y_{1}, \cdots, y_{q}, w_{1}, \cdots, w_{q}\right)=\left\{\gamma \in \Delta \mid \gamma\left(w_{j}\right)=y_{j}, 1 \leqq j \leqq q\right\}$. By Lemma 4.1, there is an $x_{1} \in V_{1}$ such that for all $y_{1} \in \mathbf{T}_{(a)}, \Delta\left(y_{1}, x_{1}\right)$ is infinite.

Let $r \in\{1,2, \cdots, n-1\}$ and suppose that $x_{j} \in V_{j}(1 \leqq j \leqq r)$ have been found with the property that for all $y_{1}, \cdots, y_{r} \in \mathbf{T}_{(a)}, \Delta\left(y_{1}, \cdots, y_{r}\right.$, $\left.x_{1}, \cdots, x_{r}\right)$ is infinite. Fixing $\left(y_{1}, \cdots, y_{r}\right) \in \mathbf{T}_{(a)}^{r}$ and applying Lemma 4.1 with $\Delta\left(y_{1}, \cdots, y_{r}, x_{1}, \cdots, x_{r}\right)$ in place of $\Delta$, we find that $m$-almost 
all $x \in V_{r+1}$ have the property that for all $y_{r+1} \in \mathbf{T}_{(a)}, \Delta\left(y_{1}, \cdots, y_{r+1}\right.$, $\left.x_{1}, \cdots, x_{r}, x\right)$ is infinite. Hence, $m$-almost all $x \in V_{r+1}$ have the property that for all $y_{1}, \cdots, y_{r+1} \in \mathbf{T}_{(a)}, \Delta\left(y_{1}, \cdots, y_{r+1}, x_{1}, \cdots, x_{r}, x\right)$ is infinite. In particular, an $x_{r+1} \in V_{r+1}$ with this property exists.

Hence, by induction, there are $x_{j} \in V_{j}(1 \leqq j \leqq n)$ such that for all $y_{1}, \cdots, y_{n} \in \mathbf{T}_{(a)}, \Delta\left(y_{1}, \cdots, y_{n}, x_{1}, \cdots, x_{n}\right)$ is infinite and, in particular, nonvoid. Hence, $\left\{x_{1}, \cdots, x_{n}\right\}$ is a $K_{a, 4}$-set.

THeOREM 4.3. Let $k, G, \Delta$, and $a$ be as in Lemma 4.1. Let $G$ be metrizable. Let $U$ be a nonvoid open subset of $G$. Then $U$ contains a $K_{a, 4}$-set homeomorphic to the Cantor set.

Proof. Repeat the proof of [4, (41.5), part III], choosing all characters in $\Delta$ and using Lemma 4.2 whenever [4] uses [4, (41.4)].

REMARK 4.4. We now proceed to reduce Theorem III to the case described in Theorem 4.3.

LEMMA 4.5. Let $k$ be an integer greater than one. Let $X$ be the weak direct product of infinitely many copies of $\mathbf{T}_{(k)}$. Let $\Delta$ be an infinite subset of $X$. Then there exist an integer $a \geqq 2$ and an infinite subset $\Gamma$ of $\Delta$ with the property that whenever $\gamma_{1}$ and $\gamma_{2}$ are distinct elements of $\Gamma$, then $\gamma_{1} \gamma_{2}^{-1}$ has order exactly a.

Proof. We remark that this result is trivial if $k$ is prime. (Take $a=k$ and $\Gamma=\Delta$.)

Let $b_{0}=k$ and $\Delta_{0}=\Delta_{\text {. Let }} \gamma_{1} \in \Delta_{0}$ 。 Let $\Gamma_{1}=\left\{\gamma_{1} \alpha^{-1} \mid \alpha \in \Delta_{0}\right\}$. Since $\Gamma_{1}$ is infinite, there is an integer $b_{1}, 2 \leqq b_{1} \leqq b_{0}$, such that $\Gamma_{1}$ contains infinitely many elements of order $b_{1}$. Let $\Delta_{1}=\left\{\alpha \in \Delta_{0} \mid \gamma_{1} \alpha^{-1}\right.$ has order $\left.b_{1}\right\}$. Suppose that $n \in \mathbf{N}$ and that $\gamma_{1}, \cdots, \gamma_{n}, \Gamma_{1}, \cdots, \Gamma_{n}, b_{1} \cdots, b_{n}$ and $\Delta_{1}, \cdots, \Delta_{n}$ have been found such that for $1 \leqq j \leqq n$ we have (i) $\gamma_{j} \in$ $\Delta_{j-1}, \Gamma_{j}=\left\{\gamma_{j} \alpha^{-1} \mid \alpha \in \Delta_{j-1}\right\}, \Gamma_{j}$ has infinitely many elements of order $b_{j}$, $2 \leqq b_{j} \leqq b_{j-1}$, and $\Delta_{j}=\left\{\alpha \in \Delta_{j-1} \mid \gamma_{j} \alpha^{-1}\right.$ has order $\left.b_{j}\right\}$. Observe that from (i) it follows that (ii) for $1 \leqq j \leqq n$, we have $\gamma_{j} \notin \Delta_{j}$ so $\Delta_{j}$ is a proper infinite subset of $\Delta_{j-1}$ and the $\gamma_{j}$ are distinct.

Let $\gamma_{n+1} \in \Delta_{n}$ 。 Let $\Gamma_{n+1}=\left\{\gamma_{n+1} \alpha^{-1} \mid \alpha \in \Delta_{n}\right\}$. Since $\Gamma_{n+1}$ is infinite, there is an integer $b_{n+1}$ with $2 \leqq b_{n+1} \leqq b_{n}$ such that $\Gamma_{n+1}$ contains infinitely many elements of order $b_{n+1}$. Let $\Delta_{n+1}=\left\{\alpha \in \Delta_{n} \mid \gamma_{n+1} \alpha^{-1}\right.$ has order $\left.b_{n+1}\right\}$. Thus, we can define $\gamma_{n}, \Gamma_{n}, \Delta_{n}$, and $b_{n}$ for all $n \in \mathbf{N}$ in such a way that properties (i) hold for all $n$. Since $\left\{b_{n}\right\}$ is a monotone nonincreasing sequence of integers greater than one, there exist positive integers $r$ and $a$ such that $b_{n}=a$ for all $n>r$. Let $\Gamma=$ $\left\{\gamma_{r+n} \mid n \in \mathbf{N}\right\}$. We show that $\Gamma$ and $\alpha$ are as demanded. Let $n_{1}$ and $n_{2} \in \mathbf{N}$ with $n_{1}>n_{2}$. Then, by construction of the $\Delta_{n}$, we have $\gamma_{r+n_{1}} \in$ 
$\Delta_{r+n_{1}-1} \subset \Delta_{r+n_{2}}$ so $\gamma_{r+n_{2}} \gamma_{r+n_{1}}^{-1}$ has order $b_{r+n_{2}}=a$.

LEMma 4.6. Let $k$ be an integer greater than one. Let $I$ be an infinite index set and let $X=\prod_{i \in I}^{*} G_{\iota}$, where each $G_{c}$ is a copy of $\mathbf{T}_{(k)}$. Let $\Delta$ be an infinite subset of $X$. Then there exist an integer $a \geqq 2$ and an infinite subset $\Delta_{0}$ of $\Delta$ and a finite (possibly empty) subset $I_{0}$ of $I$ such that projection of $\Delta_{0}$ onto $Y=\prod_{\imath \in I \backslash I_{0}}^{*} G_{\iota}$ gives an infinite subset $\widetilde{\Delta}_{0}$ of $Y$ consisting solely of elements of order $a$ and such that whenever $\gamma_{1}$ and $\gamma_{2}$ are distinct elements of $\tilde{J}_{0}, \gamma_{1} \gamma_{2}^{-1}$ has order $a$.

Proof. By Lemma 4.5, there exist an integer $a_{1} \geqq 2$ and an infinite subset $\Gamma_{1}$ of $\Delta$ such that whenever $\gamma_{1}$ and $\gamma_{2}$ are distinct elements of $\Gamma_{1}, \gamma_{1} \gamma_{2}^{-1}$ has order $\alpha_{1}$. Let $\widetilde{\Gamma}_{1}$ be an infinite subset of $\Gamma_{1}$ consisting of elements all of the same order $b_{1}$. It is clear that $b_{1} \geqq a_{1}$. (If $\gamma_{1}$ and $\gamma_{2}$ are distinct elements of $\widetilde{\Gamma}_{1}$, then $\gamma_{1} \gamma_{2}^{-1}$ has order at most $b_{1}$. But $\gamma_{1} \gamma_{2}^{-1}$ has order $a_{1}$.) If $b_{1}=a_{1}$, we are done. (Take $I_{0}=\varnothing$, $\Delta_{0}=\widetilde{\Gamma}_{1}$, and $a=a_{1}$ ) Suppose $b_{1}>a_{1}$. Let $\widetilde{\gamma}_{1} \in \widetilde{\Gamma}_{1}$. There is a finite subset $I_{1}$ of $I$ such that the cth coordinate of $\tilde{\gamma}_{1}$ is the identity of $G_{\iota}$ for $\iota \notin I_{1}$. Let $X_{1}=\prod_{\iota \bullet I \backslash I_{1}}^{*} G_{\iota}$ 。 Since $I_{1}$ is finite and $\widetilde{\Gamma}_{1}$ is infinite, projection of $\widetilde{\Gamma}_{1}$ onto $X_{1}$ (denoted by $\pi_{1}$ ) gives an infinite subset $\Delta_{1}$ of $X_{1}$ consisting of elements of order at most $a_{1}$. (For $\alpha \in \widetilde{\Gamma}_{1}$, order of $\pi_{1}(\alpha)$ in $X_{1}=$ order of $\pi_{1}\left(\alpha \tilde{\gamma}_{1}^{-1}\right)$ in $X_{1} \leqq \alpha_{1}$ ) Applying Lemma 4.5 to $X_{1}$ and $\Delta_{1}$ we get an integer $a_{2}$ with $2 \leqq a_{2} \leqq a_{1}$ and an infinite subset $\Gamma_{2}$ of $\Delta_{1}$ such that whenever $\gamma_{1}$ and $\gamma_{2}$ are distinct elements of $\Gamma_{2}$, then $\gamma_{1} \gamma_{2}^{-1}$ has order $a_{2}$. Let $\widetilde{\Gamma}_{2}$ be an infinite subset of $\Gamma_{2}$ consisting of elements all of the same order $b_{2}$ 。 Then we have $a_{2} \leqq b_{2} \leqq a_{1}<b_{1}$. If $a_{2}=b_{2}$, we are done. (Take $I_{0}=I_{1}, a=a_{2}, Y=X_{1}$, and $\Delta_{0}=$ $\left\{\alpha \in \Delta \mid \pi_{1}(\alpha) \in \widetilde{\Gamma}_{2}\right) \quad$ Suppose $a_{2}<b_{2} \leqq a_{1}<b_{1}$. Pick $\widetilde{\gamma}_{2} \in \widetilde{\Gamma}_{2}$; let $I_{2}=$ $\left\{c \in I \backslash I_{1} \mid\right.$ cth coordinate of $\widetilde{\gamma}_{2}$ is not the identity of $\left.G_{c}\right\}$; project $\widetilde{\Gamma}_{2}$ onto $X_{2}=\prod_{\left.\iota \in \backslash \backslash I_{1} \cup I_{2}\right)}^{*} G_{c} ; \cdots$ etc. We must eventually have $b_{n}=a_{n}$ for some $n$ (otherwise, $\left\{b_{n}\right\}$ would be an infinite strictly decreasing sequence of positive integers). For that $n$, we have a finite subset $I_{0}=I_{1} \cup \cdots \cup I_{n-1}$ of $I$ and an infinite subset $\widetilde{\Gamma}_{n}$ of $Y=\prod_{\iota \in I \backslash I_{0}}^{*} G_{\iota}$ such that all elements of $\widetilde{\Gamma}_{n}$ have order $a_{n}=b_{n}$ and such that whenever $\gamma_{1}$ and $\gamma_{2}$ are distinct elements of $\widetilde{\Gamma}_{n}, \gamma_{1} \gamma_{2}^{-1}$ has order $a_{n}$. Let $\Delta_{0}=\left\{\alpha \in \Delta \mid \pi(\alpha) \in \widetilde{\Gamma}_{n}\right)$, where $\pi$ is the projection of $X$ onto $Y$.

THEOREM 4.7. Let $k$ be an integer greater than one. Let $G=$ $\prod_{c \in I} G_{c}$, where each $G_{\iota}$ is a copy of $\mathbf{T}_{(k)}$ and $I$ is infinite. Let $\Delta$ be an infinite subset of $X$. Then there is an integer a greater than one such that every neighborhood of the identity of $G$ contains a $K_{a,\lrcorner}$-set homeomorphic to the Cantor set. 
Proof. We may suppose that $\Delta$ is countable. We identify $X$ with $\prod_{\iota \in I}^{*} G_{\iota}$. Let $a, I_{0}, Y$, and $\widetilde{\Delta}_{0}$ be as in Lemma 4.6. Let $I_{1}=$ $\left\{\iota \in I \backslash I_{0} \mid\right.$ some $\gamma \in \hat{\Delta}_{0}$ has $\iota$ th coordinate different from the identity of $\left.G_{c}\right\}$. Plainly $I_{1}$ is countably infinite. Let $I_{2}=I \backslash\left(I_{0} \cup I_{1}\right)$. Let $G_{j}=$ $\Pi_{\iota \in I_{j}} G_{\iota}$, and let $G_{j}$ have character group $X_{j}, j=0,1,2$. Since $I_{1}$ is countable, $G_{1}$ is metrizable. Since $I_{0}$ is finite, $G_{0}$ is finite. Let $\Gamma_{0}$ be the image of the projection of $\tilde{\Delta}_{0}$ onto $X_{1}$. We may suppose that our neighborhood of the identity of $G$ has the form $U=\left\{e_{0}\right\} \times V_{1} \times V_{2}$, where $e_{0}$ is the identity of $G_{0}$ and $V_{j}$ is open in $G_{j}, j=1,2$. Applying Theorem 4.3 to $k, G_{1}, \Gamma_{0}$, and $a$, we find a subset $P_{1}$ of $V_{1}$ homeomorphic to the Cantor set which is a $K_{a, \Gamma_{0}}$-set. Let $P=\left\{e_{0}\right\} \times P_{1} \times\left\{e_{2}\right\}$, where $e_{2}$ is the identity of $G_{2}$. Then $P$ is a $K_{a, 4}$-set in $U$ homeomorphic to the Cantor set.

Proof of Theorem III. 4.8. If $G$ is a compact torsion group, then there are integers $r_{1}, \cdots, r_{q}$ greater than one and disjoint infinite index sets $I_{1}, \cdots, I_{q}$ and there is a finite abelian group $F$ such that $G$ is topologically isomorphic to $F \times G_{1} \times \cdots \times G_{q}$, where $G_{j}=\prod_{\iota \in I_{j}} K_{\iota}$ and each $K_{\iota}$ is a copy of $\mathbf{T}_{\left(r_{j}\right)}$ when $\iota \in I_{j}(1 \leqq j \leqq q)$. Let $G_{j}$ have character group $X_{j}(1 \leqq j \leqq q)$. Then for some $j_{0}$, the image $\Gamma$ of the projection of $\Delta$ onto $X_{j_{0}}$ is infinite. Let $a$ be as in Theorem 4.7 applied to $G_{j_{0}}, X_{j_{0}}$, and $\Gamma$. Let $U$ be a neighborhood of the identity of $G$. We will prove that $U$ contains a $K_{a, 4}$-set homeomorphic to the Cantor set. Clearly, this will establish Theorem III. We may suppose that $U$ has the form $\left\{e_{F}\right\} \times U_{1} \times \cdots \times U_{q}$, where $e_{F}$ is the identity of $F$ and $U_{j}$ is a neighborhood of the identity $e_{j}$ of $G_{j}(1 \leqq j \leqq q)$. By Theorem 4.7, $U_{j_{0}}$ contains a $K_{a, r^{-}}$-set $P_{j_{0}}$ homeomorphic to the Cantor set. Let

$$
P=\left\{e_{F}\right\} \times\left\{e_{1}\right\} \times \cdots \times\left\{e_{j_{0}-1}\right\} \times P_{j_{0}} \times\left\{e_{j_{0}+1}\right\} \times \cdots \times\left\{e_{q}\right\} .
$$

Then $P$ is a $K_{a, 4}$-set in $U$ homeomorphic to the Cantor set.

\section{Examples.}

5.1. The hypothesis that $\bar{\Delta}$ is not compact is necessary in Theorem II. If $\bar{\Delta}$ is compact, then there is a nonempty open $U \subset G$ which contains no $K_{0,4}$-set and no $K_{a, 4}$-set for any integer $a \geqq 2$. Indeed, let $U=\{x \in G:|\gamma(x)-1|<1$ for all $\gamma \in \bar{\Delta}\}$. Then $U$ is an open neighborhood of the identity in $G$ and $\operatorname{Re} \gamma(x)>0$ for all $x \in U$ and all $\gamma \in \Delta$. Hence, the function -1 cannot be matched within 1 on any nonvoid subset of $U$ by any $\gamma \in \Delta$, nor can the function $\omega_{a}$ (where $\omega_{a}$ is an $a$ th root of unity with $\operatorname{Re} \omega_{a}<0$ ) be matched on any nonvoid subset of $U$ by any $\gamma \in \Delta$ for any integer $a \geqq 2$. Hence, no subset of $U$ is a $K_{0,4}$-set or a $K_{a, 4}$-set. 
5.2. The phrase "a translate of" is a necessary part of the conclusion of Theorem III, as is shown by the following example. Let $G=\mathbf{T}_{(2)} \times H$, where $H$ is the product of infinitely many copies of $\mathbf{T}_{(3)}$. Write $X=\mathbf{Z}_{2} \times Y$, where $Y$ is the character group of $H$. Let $\Delta=\{1\} \times Y$. Let $U=\{-1\} \times H$. Then $U$ is open in $G$ and $\gamma(x) \in-\mathbf{T}_{(3)}$ for all $x \in U$ and all $\gamma \in \Delta$, so the constant function 1 cannot be matched on any subset of $U$ by any $\gamma \in \Delta$. Hence, no subset of $U$ is a $K_{a, 4}$-set for any integer $a \geqq 2$.

5.3. The hypothesis that $G$ is a compact torsion group in Theorem III cannot be weakened to the hypothesis that $G$ is compactly generated and contains a compact open torsion subgroup. For example, let $H$ be an infinite compact torsion group and let $G=\mathbf{Z} \times H$. Take $\Delta=\mathbf{T} \times\{e\}$ (where $e$ is the identity of the character group of $H$ ) and $U=\{0\} \times H$. Then $\gamma(x)=1$ for all $x \in U$ and all $\gamma \in \Delta$. Hence, whenever $P \subset G$ is such that a translate of $P$ is contained in $U$, we have $\gamma$ constant on $P$. Therefore, no such totally disconnected $P$ containing more than one point can be a $K_{a, 4}$-set for any integer $a \geqq 2$.

5.4. The hypothesis of local connectedness or something closely related to connectedness (cf. Theorem 2.1) in Theorems II and I respectively cannot be weakened to the hypothesis that $G$ is not a torsion group. Indeed, there exist a compact metrizable group $G$ which is not a torsion group and an infinite subset $\Delta$ of $X$ such that $G$ contains no $K_{0,4}$-set. For example, let $G=\prod_{j=2}^{\infty} \mathbf{T}_{(2 j)}$. Then, writing $X=\prod_{j=2}^{* \infty} \mathbf{Z}_{2 j}$ and letting $\Delta=\left\{\gamma_{2}, \gamma_{3}, \cdots\right\}$ where $\gamma_{j}$ has $j$ th coordinate equal to $j$ and the rest zero, we have $\gamma_{j}(x)= \pm 1$ for all $x \in G$ and all $j$, so every nonempty subset of $G$ fails to be a $K_{0,4}$-set.

Also, there exist a compact metrizable group $G$ which is not a torsion group and an infinite subset $\Delta$ of $X$ such that no subset of $G$ containing more than one point is a $K_{a, 5}$ set for any integer $a \geqq 2$. Let $G=\prod_{j=1}^{\infty} \mathbf{T}_{\left(p_{j}\right)}$ where $p_{j}$ is the $j$ th prime. Write $X=\prod_{j=2}^{* \infty} \mathbf{Z}_{p_{j}}$ and let $\Delta=\left\{\gamma_{1}, \gamma_{2}, \cdots\right\}$ where $\gamma_{j}$ has $j$ th coordinate equal to 1 and the rest zero. Let $P$ be a subset of $G$ containing at least two points. Let $a \geqq 2$ be an integer. We will show that $P$ is not a $K_{a, 4}$-set. Let $p_{k}$ be a divisor of $a$. The open-closed sets in $G$ form a basis for the topology of $G$, so there are two distinct $\mathbf{T}_{\left(p_{k}\right)}$-valued (and, hence, $\mathbf{T}_{(a)}$-valued) continuous functions, $f_{1}$ and $f_{2}$, on $P$ both different from 1. If either $f_{i}$ is matched on $P$ by some $\gamma_{j}$, it must be matched by $\gamma_{k}$ since no other $\gamma_{j}$ attains values in $\mathbf{T}_{\left(p_{k}\right)}$ different from 1 . Thus either $f_{1}$ or $f_{2}$ is a $\mathbf{T}_{(a)}$-valued continuous function not matched on $P$ by any $\gamma_{j}$. Hence, $P$ is not a $K_{a, 4}$-set. 


\section{REFERENCES}

1. J. Dugundji, Topology, Allyn and Bacon, Inc., Boston, 1966.

2. B. Eckmann, Über monothetische Gruppen, Comment. Math. Helv. 16 (1943-1944), 249-263.

3. G. Helmberg, A theorem on equidistribution in compact groups, Pacific J. Math., 8 (1958), 227-241.

4. E. Hewitt and K. A. Ross, Abstract Harmonic Analysis, Volume II, Die Grundlehren der mathematischen Wissenschaften in Einzeldarstellungen, Band 152, Springer-Verlag, New York, 1970.

5. E. Hlawka, Zur formalen Theorie der Gleichvarteilung in kompakton Gruppen, Rend. Circ. Mat. Palermo (2) 4 (1955), 33-47.

6. Y. Katznelson, An Introduction to Harmonic Analysis, John Wiley and Sons, Inc., New York, 1968.

7. R. Kaufman, A functional method for linear sets, Israel J. Math., 5 (1967). 185-187.

8. W. Rudin, Fourizr Analysis on Groups, Interscience tracts in pure and applied mathematics, No. 12, John Wiley and Sons, Inc., New York, 1962.

9. H. Weyl, Uoor dise Gleichverteilung von Zainlen mod. Eins, Math. Ann. 77 (1916), $313-352$.

Received August 25, 1971 and in revised form April 25, 1972. The author was a National Science Foundation Graduate Fellow, 1969-1971. The results in this paper are taken from the author's doctoral dissertation, which was written at the University of Washington under the direction of Professor Edwin Hewitt.

UNIVERSITY OF WASHINGTON

Present address: California State College, Dominguez Hills, 90747 


\section{PACIFIC JOURNAL OF MATHEMATICS}

\section{EDITORS}

H. SAMELSON

Stanford University

Stanford, California 94305

C. R. Hовву

University of Washington

Seattle, Washington 98105
J. DugundJI

Department of Mathematics University of Southern California

Los Angeles, California 90007

RICHARD ARENS

University of California

Los Angeles, California 90024

\section{ASSOCIATE EDITORS}

E. F. BECKENBACH

B. H. NeumanN

F. WOLF

K. YoSHIDA

\section{SUPPORTING INSTITUTIONS}

UNIVERSITY OF BRITISH COLUMBIA

CALIFORNIA INSTITUTE OF TECHNOLOGY

UNIVERSITY OF CALIFORNIA

MONTANA STATE UNIVERSITY

UNIVERSITY OF NEVADA

NEW MEXICO STATE UNIVERSITY

OREGON STATE UNIVERSITY

UNIVERSITY OF OREGON

OSAKA UNIVERSITY
UNIVERSITY OF SOUTHERN CALIFORNIA

STANFORD UNIVERSITY

UNIVERSITY OF TOKYO

UNIVERSITY OF UTAH

WASHINGTON STATE UNIVERSITY

UNIVERSITY OF WASHINGTON

AMERICAN MATHEMATICAL SOCIETY

NAVAL WEAPONS CENTER

Printed in Japan by International Academic Printing Co., Ltd., Tokyo, Japan 


\section{Pacific Journal of Mathematics}

\section{Vol. 44, No. $1 \quad$ May, 1973}

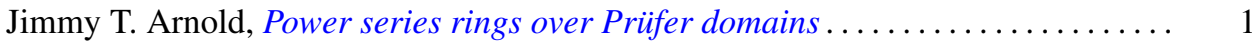

Maynard G. Arsove, On the behavior of Pincherle basis functions . . . . . . . . . 13

Jan William Auer, Fiber integration in smooth bundles ................. 33

George Bachman, Edward Beckenstein and Lawrence Narici, Function algebras

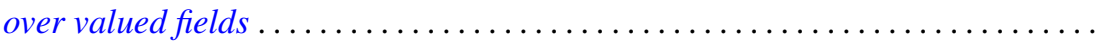

Gerald A. Beer, The index of convexity and the visibility function . . . . . . . . . . .

James Robert Boone, A note on mesocompact and sequentially mesocompact

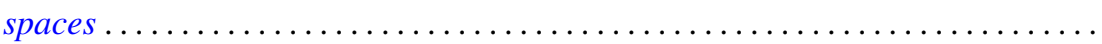

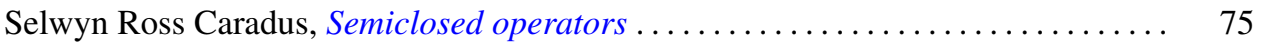

John H. E. Cohn, Two primary factor inequalities . . . . . . . . . . . . . . . 81

Mani Gagrat and Somashekhar Amrith Naimpally, Proximity approach to

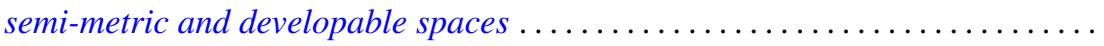

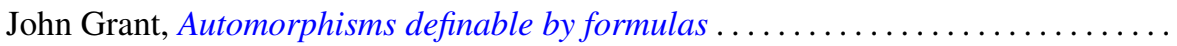

Walter Kurt Hayman, Differential inequalities and local valency ..............

Wolfgang H. Heil, Testing 3-manifolds for projective planes . . . . . . . . . . . . .

107

Melvin Hochster and Louis Jackson Ratliff, Jr., Five theorems on Macaulay

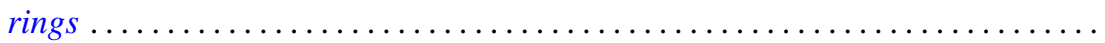

Thomas Benton Hoover, Operator algebras with reducing invariant subspaces ....

James Edgar Keesling, Topological groups whose underlying spaces are separable

Fréchet manifolds...

Frank Leroy Knowles, Idempotents in the boundary of a Lie group . .

191

George Edward Lang, The evaluation map and EHP sequences ...

201

Everette Lee May, Jr, Localizing the spectrum . . . . . . . . . . . .

211

Frank Belsley Miles, Existence of special $K$-sets in certain locally compact abelian groups.

Susan Montgomery, A generalization of a theorem of Jacobson. II . .

T. S. Motzkin and J. L. Walsh, Equilibrium of inverse-distance forces in

three-dimensions.

Arunava Mukherjea and Nicolas A. Tserpes, Invariant measures and the converse

of Haar's theorem on semitopological semigroups .

James Waring Noonan, On close-to-convex functions of order $\beta$

Donald Steven Passman, The Jacobian of a growth transformation

Dean Blackburn Priest, A mean Stieltjes type integral ........ .

Joe Bill Rhodes, Decomposition of semilattices with applications to topological

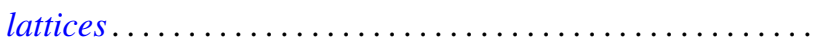

Claus M. Ringel, Socle conditions for $\mathrm{QF}-1$ rings ..........

Richard Rochberg, Linear maps of the disk algebra

Roy W. Ryden, Groups of arithmetic functions under Dirichlet convolution . .

Michael J. Sharpe, A class of operators on excessive functions

Erling Stormer, Automorphisms and equivalence in von Neumann algebras ..

Philip C. Tonne, Matrix representations for linear transformations on series

analytic in the unit disc. 